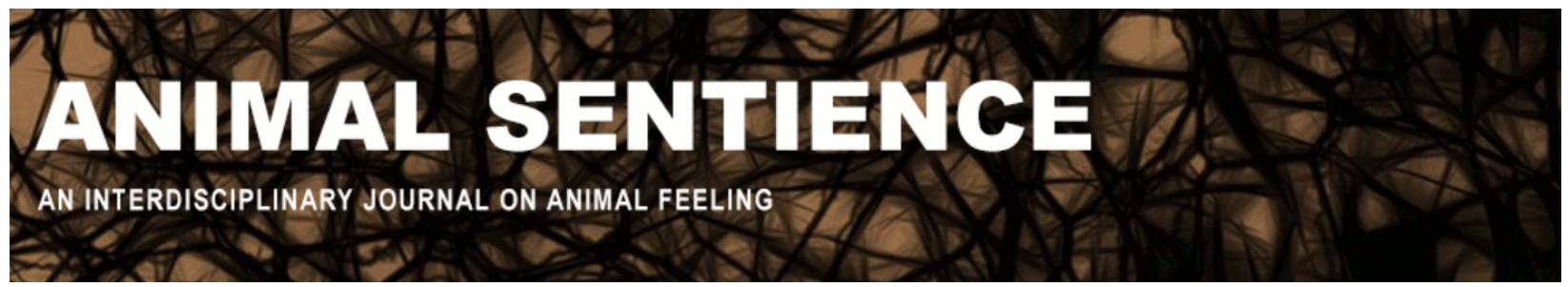

Walters, Edgar T. (2018) Defining pain and painful sentience in animals. Animal Sentience 21(14)

DOI: $10.51291 / 2377-7478.1360$

Date of submission: 2018-07-22

Date of acceptance: 2018-07-26

(c)

This article has appeared in the journal Animal

Sentience, a peer-reviewed journal on animal

cognition and feeling. It has been made open access,

free for all, by WellBeing International and deposited

in the WBI Studies Repository. For more information,

please contact

wbisr-info@wellbeingintl.org.

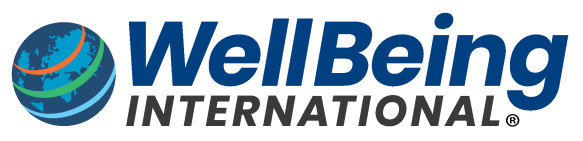

SOLUTIONS FOR PEOPLE, ANIMALS AND ENVIRONMENT 


\title{
Defining pain and painful sentience in animals
}

\author{
Commentary on Sneddon et al. on Sentience Denial
}

\author{
Edgar T. Walters \\ Department of Integrative Biology and Pharmacology \\ McGovern Medical School, University of Texas Health Science Center at Houston
}

\begin{abstract}
Sentience is essential to most definitions of pain, including a detailed definition invoked by Sneddon et al. to argue that adult and perhaps larval fish feel pain. Because proving painful sentience in non-human animals is not feasible, multiple lines of indirect evidence are needed to implicate pain. This commentary examines the list of 17 criteria used by Sneddon et al. to conclude that fish have conscious pain. The criteria include tests of nociceptive, motivational, and cognitive properties useful for revealing pain-like states that can be understood biologically and be related evolutionarily to human pain. However, additional research is needed to define the crucial aversive component of pain-like states in fish.
\end{abstract}

Edgar T. Walters is Professor of Integrative Biology and Pharmacology and holder of the Fondren Chair in Cellular Signaling. His research has focused on long-term alterations of neural and behavioral responses to peripheral injury in molluscs (Aplysia and squid), on mechanisms of persistent pain induced by spinal cord injury or peripheral injury in rodents, and on comparative approaches to understanding the evolution of pain-related mechanisms. Website

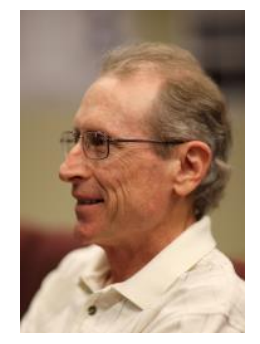

Arguments for the likelihood that fish feel pain are included in Sneddon et al.'s (2018) target article, continuing a heated debate that has engaged many readers of Animal Sentience since its inception. Crucial to arguments on all sides of this debate is how pain is defined. In arguing for painful sentience in adult and perhaps larval fish, the target article invokes an unusually detailed definition of animal pain (Sneddon et al., 2014), which is widely cited. My commentary addresses the logic behind this definition and the scientific evidence that it and similar definitions require for the strong implication of pain in non-human species.

Pain Definitions and Sentience. For most people, the word "pain" has many meanings, but conscious suffering is central to the human pain experience. This sentient aspect of pain is assumed in the title of the target article. This is also the view of the International Association for the Study of Pain (IASP, 1994), which defines pain as "an unpleasant sensory and emotional experience associated with actual or potential tissue damage, or described in terms of such damage." This definition represents a clinical perspective on pain, which is evident in its inclusion of verbal description as a sufficient indicator of pain. Verbal self-report is unavailable in nonhuman species, but the remainder of the definition is applicable to the debate about whether fish and other animals feel pain. Most important, the term "unpleasant" implies that an individual in pain is sentient and, if the pain is sufficient, that the individual suffers. 
Most definitions of pain distinguish two components: (1) a sensory component that enables an organism to detect and to respond quickly and selectively to those stimuli that cause or threaten tissue damage (nociception), and (2) an emotional component that motivates adaptive behavior during and after a noxious event. The nociceptive function is not controversial, being demonstrated in all animal taxa that have been tested experimentally, including fish (Sneddon et al., 2014; 2018) and diverse invertebrates (Walters, 2018). It is the conscious emotional (affective) reactions to noxious stimulation that are contentious. Demonstrating suffering or any other form of sentience in an organism is a notoriously difficult problem (e.g., Harnad, 2016). Investigators of animal pain usually sidestep this other-minds problem by addressing the aversiveness (defined operationally) rather than unpleasantness (defined subjectively) of putative pain experience. The word "aversion" comes from the Latin word meaning to turn away from, and it is measured objectively as avoidance by an animal of contexts or behaviors associated with noxious events (e.g., conditioned avoidance of a place where noxious stimulation occurs, or conditioned leg flexion when leg extension is punished). Such avoidance shows that the noxious event produces a negative motivational state. Aversion also implies that the motivational state has the same protective function - avoidance of further injury or stress - that is implicit in the IASP definition of conscious pain.

Sneddon et al.'s criteria for animal pain. The practical impossibility of proving painful sentience in animals is often acknowledged by both sides of the fish pain debate. Instead, multiple lines of evidence are sought to "triangulate" the likely existence of a pain state that cannot be measured directly (Bateson, 1991; Sneddon et al., 2014). Sneddon (2009) proposed that pain in animals is "the perception and aversive sensory experience of a noxious stimulus associated with potential or actual injury." This definition was greatly expanded by Sneddon et al. (2014), who listed 17 specific criteria, suggesting that, taken together, these can provide overwhelming evidence for animal pain. The 17 criteria were based on plausible assumptions about protective functions of pain that have long been considered (e.g., Bateson, 1991; Wall, 1979; Walters, 1994) and on correlates of pain experience in humans. Below, I comment briefly on each of the 17 criteria proposed as evidence for pain in fish, using the numbers listed in Table 2 of Sneddon et al. (2014).

Nociceptors (1), Physiological responses (5), Behavioral changes from norm (7). All the widely used definitions of pain have a link to actual or imminent tissue damage, so all require mechanisms to detect noxious stimuli. Primary nociceptors tuned to damaging stimuli (criterion 1) have been described in diverse invertebrate and vertebrate species, including fish (Sneddon et al., 2003), indicating the biological value of nociception (Walters, 2018). If imminent tissue damage is biologically significant, it is to be expected that nociceptor activity will trigger physiological and behavioral responses that differ from those observed in the absence of noxious stimulation (criteria 5 and 7). Fulfillment of these three criteria indicates the presence of nociception, which is a necessary but not sufficient condition for evoked pain under normal conditions (although not during allodynia, or for spontaneous pain). 


\section{Seventeen (17) Criteria for Pain Perception \\ (Adapted from Sneddon et al., 2014, Table 2) \\ Criterion met by at least one species of: \\ Mammals, Birds, Amphibians/reptiles, Fish, Cephalopods, Decapods, Insects}

(1) Nociceptors MBAFCDI

(2) Pathways to CNS MBAFCDI

(3) Central processing in brain MBAFCDI

(4) Receptors for analgesic drugs MBAFCD

(5) Physiological responses MBAFCD

(6) Movement away from noxious stimuli MBAFCDI

(7) Behavioral changes from norm MBAFCDI

(8) Protective behavior MBAFCD

(9) Responses reduced by analgesic drugs MBAFCDI

(10) Self-administration of analgesia MBF

(11) Responses with high priority over other stimuli MFCD

(12) Pay cost to access analgesia MBF

(13) Altered behavioral choices/preferences MBFCDI

(14) Relief learning MBI

(15) Rubbing, limping or guarding MBFCD

(16) Paying a cost to avoid stimulus MBFD

(17) Trade-offs with other requirements MBFD

Movement away from noxious stimuli (6); Protective behavior (8); Responses with high priority over other stimuli (11); Rubbing, limping, or guarding (15). These criteria all refer to the protective and recuperative functions of behaviors evoked immediately or with a delay by noxious stimuli. Protective and recuperative behaviors of some kind are required by definitions of pain based upon its presumed biological functions. Across the animal kingdom, immediate defensive behaviors elicited by noxious stimuli often include escape and/or withdrawal (criteria 6 and 8), while delayed recuperative behaviors sometimes include grooming and/or guarding (criterion 15), all of which take priority over behaviors (criterion 11) that could interfere with defense and recuperation during and after noxious stimulation (Walters, 1994). Thus, these functional criteria are useful for demonstrating both immediate nociception and sensitized recuperative states that may be analogous to long-lasting pain states in humans that follow serious injury (Walters, 2012). Controlled studies of immediate or persistent pain-like behavior following relatively severe injury in fish have not been reported, although anecdotal observations of a lack of persistent pain-like behavior after surgical procedures have been described (Rose et al., 2012).

Pathways to CNS (2), Central Processing in brain (3). These criteria are based on an analogy to human pain, the conscious experience of which is assumed by virtually all scientists to occur in the brain (with disagreement about which parts are involved; e.g., see Devor, 2016). There is, however, no logical requirement that painful sentience be localized to a central brain. The invertebrate with the largest nervous system, and that is often suggested as most likely to experience pain - the octopus - has most of its neurons distributed peripherally in numerous, complex ganglia in each of its arms rather than its central brain. The possibility exists that each octopus arm might be independently capable of feeling pain, just as an isolated arm is capable of complex behaviors after removal from the rest of the octopus. Meeting criteria 2 and 3 is logically 
unnecessary for implicating pain. Central processing does suggest integration of nociceptive information with other information. Because it has been found in even the simplest animal species examined neurophysiologically, central processing of nociceptive information by itself probably says little about whether conscious pain is likely.

Receptors for analgesic drugs (4), Responses reduced by analgesic drugs (9), Self-administration of analgesia (10). The existence in an animal of systems that suppress nociceptive responses when stimulated by analgesics would seem, prima facie, to provide strong evidence for pain based on analogy to human pain systems. However, "analgesic" is defined ultimately on the basis of verbal reports of pain suppression in humans, so we can't be sure that any drug is actually an analgesic in non-human animals, especially in species distantly related to humans. Furthermore, all analgesic treatments have multiple effects. For example, opioids can have strong actions on gastrointestinal, respiratory, and inflammatory functions, any of which could affect behavioral measures of pain-like states. As a specific illustration, morphine can stimulate a toll-like receptor (TLR4), producing paradoxical effects on pain in rodents (Grace et al., 2016). TLR4 activation also promotes sickness behavior. This raises the possibility that in some species an effect of morphine on behavior interpreted as analgesic might instead reflect sickness behavior. Even when analgesic effects occur, opioids and other drugs are likely to produce analgesia at least in part by inhibiting nociceptive signaling at primary afferent and spinal levels, before it reaches brain systems where conscious pain may be experienced (by human analogy). This limits the implications to be drawn about painful sentience. Furthermore, some analgesics (notably opioids) are intrinsically rewarding in many species, perhaps including fish (Brock et al., 2017), so observation of selfadministration may reflect a motivation quite different from a drive to obtain analgesia. Thus, the design and interpretation of experiments using human analgesics to implicate pain in other animals is quite challenging. Nonetheless, careful fulfillment of these three criteria can provide useful evidence that an animal has a nociceptive system similar to systems that suppress nociception and pain in humans.

\footnotetext{
Altered behavioral choices/preferences (13), Relief learning (14), Paying a cost to avoid stimulus (16), Trade-offs with other requirements (17). This set of criteria is particularly important for demonstrating higher-order properties of a potential pain state: specifically, its aversiveness (negative motivational properties) and its link to cognitive processing. Altered behavioral choices/preferences (criterion 13) refers to operant (apparently voluntary) choices made by an organism to avoid the recurrence of a noxious event. In mammalian pain studies and at least one fish study (Dunlop et al., 2006), this choice has been demonstrated by operant tests showing conditioned place avoidance (CPA) - an animal avoiding a place (or sometimes a simple cue) associated with previous noxious stimulation (electric shock in the fish study, which may activate nociceptors, but might also produce aversive effects independent of nociception). Conversely, another operant test, conditioned place preference (CPP) is beginning to be used in mammalian pain models to show that an animal will choose to spend time in a place where it previously received relief from ongoing pain (Navratilova, 2013). Analgesia-dependent CPP has not yet been shown in fish, although a potentially similar effect has been suggested by observations that zebrafish given noxious chemical stimulation increased the time they spent in a chamber containing a local anesthetic, lidocaine (Sneddon, unpublished data; see Sneddon, 2013).
} 
Because lidocaine is a nonspecific $\mathrm{Na}^{+}$channel blocker, it has many effects in addition to reducing nociception, which are difficult to exclude as contributing factors. For example, the noxious stimulus might have increased entry into the innately non-preferred chamber containing lidocaine, which could have reduced the ability of the fish to then leave by effects on motor pathways or other sensory systems (rather than altering cognitive preference). These unpublished results encourage the application of conventional CPP tests to fish. A major advantage of the CPP paradigm is that the analgesic is absent when the animal demonstrates its preference for the place where it remembers receiving analgesia, eliminating the possibility of acute pharmacological complications. Relief learning (criterion 14) is similar to CPP, but it is typically defined as learning that a cue predicts the termination of a noxious stimulus, usually electric shock (as opposed to learning that a place provides relief from an ongoing aversive state).

Criteria 13 and 14 both indicate motivational and cognitive contributions to states produced by noxious stimulation, and they can thus provide useful evidence for the presence of pain-like states. Whereas CPA has been reported in fish, there appears to be little evidence yet for analgesic-induced CPP or pain relief learning by fish, which could provide convincing evidence for a negative motivational state. Because a negative state thus revealed might not be pain (avoidance behavior can also be driven by states such as itch, illness, and disruptions of physiological homeostasis), a pain-like aversive state needs to be linked to noxious stimuli producing either actual injury, threat of imminent tissue damage, or demonstrable activation of nociceptors. Paying a cost to avoid a noxious stimulus and showing trade-offs with other requirements (criteria 16 and 17 ) reveal the degree of aversiveness (motivational strength) of a pain-like state in competition with other motivations. Thus, these last two criteria can further strengthen the motivational-cognitive evidence for pain-like states obtained with tests such as CPA, CPP, and pain-relief learning. In sum, motivational alterations produced by noxious events (criteria 13, 14, 16, 17, and also 10) can provide strong evidence for the existence of pain-like motivational states in non-human animals. More information is needed in fish about the aversiveness of internal states induced by noxious stimulation.

Conclusions. Definitions of animal pain based on functional assumptions as well as analogies to human pain continue to be useful. Because of the difficult problem of proving painful sentience, marshalling multiple lines of indirect evidence is the only practical strategy for implicating pain in non-human animals. The specific criteria for animal pain listed by Sneddon et al. (2014) and invoked in the target article (Sneddon et al., 2018) represent a laudable early step for systematic efforts to collect information that might strongly implicate a capacity for suffering in diverse species, including fish. The 17 proposed criteria are reasonable but not equally informative. They include tests of nociceptive, motivational, and cognitive properties that appear essential for revealing pain-like states that can be understood biologically and be related evolutionarily to human pain. Consideration of the evidence presented for these criteria suggests that additional information should be sought to define the aversive component of pain-like states in fish.

Acknowledgements. Thanks to Dr. Stevan Harnad for adding the table. Some of the ideas presented here were stimulated by ongoing research supported by National Institute of Neurological Diseases and Stroke Grant NS091759. 


\section{References}

Bateson, P. (1991). Assessment of pain in animals. Animal Behaviour 42, 827-839.

Brock, A. J., Goody, S. M. G., Mead, A. N., Sudwarts, A., Parker, M. O., and Brennan, C. H. (2017).

Assessing the value of the zebrafish conditioned place preference model for predicting human abuse potential. The Journal of Pharmacology and Experimental Therapeutics 363, 66-79.

Devor, M. (2016). Where is pain in the brain? Animal Sentience 3(34).

Dunlop, R., Millsopp, S., and Laming, P. (2006). Avoidance learning in goldfish (Carassius auratus) and trout (Oncorhynchus mykiss) and implications for pain perception. Applied Animal Behaviour Science 97, 255-271.

Grace, P. M., Strand, K. A., Galer, E. L., Urban, D. J., Wang, X., Baratta, M. V., Fabisiak, T. J., Anderson, N. D., Cheng, K., Greene, L. I., Berkelhammer, D., Zhang, Y., Ellis, A. L., Yin, H. H., Campeau, S., Rice, K. C., Roth, B. L., Maier, S. F., and Watkins, L. R. (2016). Morphine paradoxically prolongs neuropathic pain in rats by amplifying spinal NLRP3 inflammasome activation. Proceedings of the National Academy of Sciences of the United States of America 113, E3441-50.

Harnad, S. (2016). Animal sentience: The other-minds problem. Animal Sentience 1(1).

IASP Task Force on Taxonomy. (1994). Part III: Pain terms, a current list with definitions and notes on usage. Classification of chronic pain.

Navratilova, E., Xie, J. Y., King, T., and Porreca, F. (2013). Evaluation of reward from pain relief. Annals of the New York Academy of Sciences 1282, 1-11.

Rose, J. D., Arlinghaus, R., Cooke, S. J., Diggles, B. K., Sawynok, W., Stevens, E. D., and Wynne, C. D. (2012). Can fish really feel pain. Fish and Fisheries 15, 97-133.

Sneddon, L. U. (2009). Pain perception in fish: Indicators and endpoints. ILAR Journal 50, 338-342.

Sneddon, L. U. (2013). Do painful sensations and fear exist in fish? In Animal suffering: From science to law, international symposium, van der Kemp, T. A., and M. Lachance, eds. (Toronto, Canada: Carswell), pp. 93-112.

Sneddon, L. U., Braithwaite, V. A., and Gentle, M. J. (2003). Do fishes have nociceptors? Evidence for the evolution of a vertebrate sensory system. Proceedings of the Royal Society B: Biological Sciences 270, 1115-1121.

Sneddon, L. U., Elwood, R. W., Adamo, S. A., and Leach, M. C. (2014). Defining and assessing animal pain. Animal Behaviour 97, 201-212.

Sneddon, L. U., Lopez-Luna, J., Wolfenden, D. C. C., Leach, M. C., Valentim, A. M., Steenbergen, P. J., Bardine, N., Currie, A. D., Broom, D. M., and Brown, C. (2018). Fish sentience denial: Muddying the waters. Animal Sentience 21(1).

Wall, P. D. (1979). On the relation of injury to pain. The John J. Bonica lecture. Pain 6, 253-264.

Walters, E. T. (1994). Injury-related behavior and neuronal plasticity: An evolutionary perspective on sensitization, hyperalgesia, and analgesia. International Review of Neurobiology 36, 325-427.

Walters, E. T. (2012). Nociceptors as chronic drivers of pain and hyperreflexia after spinal cord injury: An adaptive-maladaptive hyperfunctional state hypothesis. Frontiers in Physiology 3, 309.

Walters, E. T. (2018). Nociceptive biology of molluscs and arthropods: Evolutionary clues about functions and mechanisms potentially related to pain. Frontiers in Physiology 9, 1049. 